RESEARCH LETTER - Physiology \& Biochemistry

\title{
Preferential dipeptide incorporation of Porphyromonas gingivalis mediated by proton-dependent oligopeptide transporter (Pot)
}

Yuko Ohara-Nemoto ${ }^{1, *}$, Mohammad Tanvir Sarwar ${ }^{1}$, Yu Shimoyama ${ }^{2}$, Takeshi Kobayakawa ${ }^{1}$, and Takayuki K. Nemoto $^{1}$

${ }^{1}$ Department of Oral Molecular Biology, Course of Medical and Dental Sciences, Nagasaki University Graduate School of Biomedical Sciences, Nagasaki 852-8588, Japan

${ }^{2}$ Division of Molecular Microbiology, Department of Microbiology, Iwate Medical University, 1-1-1 Idai-dori, Yahaba 028-3694, Japan

${ }^{*}$ Corresponding author: Yuko Ohara-Nemoto, Department of Oral Molecular Biology, Nagasaki University Graduate School of Biomedical Sciences, 1-7-1 Sakamoto, Nagasaki 852-8588, Japan Tel: +81-95-819-7643; Fax: +81-95-819-7642; email: oharanemoto@gmail.com

One sentence summary: Porphyromonas gingivalis preferentially incorporates dipeptides via proton-dependent oligopeptide transporter.

Keywords: dipeptide; dipeptidyl-peptidase; oligopeptide transporter; Porphyromonas gingivalis; protondependent oligopeptide transporter; serine/threonine transporter

Topics: amino acid incorporation; dipeptide production; resazurin assay 


\begin{abstract}
Multiple dipeptidyl-peptidases (DPPs) are present in the periplasmic space of Porphyromonas gingivalis, an asaccharolytic periodontopathic bacterium. Dipeptides produced by DPPs are presumed to be transported into the bacterial cells and metabolized to generate energy and cellular components. The present study aimed to identify a transporter responsible for dipeptide uptake in the bacterium. A real-time metabolic analysis demonstrated that $P$. gingivalis preferentially incorporated Gly-Xaa dipeptides, and then, single amino acids, tripeptides, and longer oligopeptides to lesser extents. Heterologous expression of the $P$. gingivalis serine/threonine transporter (SstT) (PGN_1460), oligopeptide transporter (Opt) (PGN_1518), and proton-dependent oligopeptide transporter (Pot) (PGN_0135) genes demonstrated that Escherichia coli expressing Pot exclusively incorporated Gly-Gly, while SstT managed Ser uptake and Opt was responsible for Gly-Gly-Gly uptake. Dipeptide uptake was significantly decreased in a $P$. gingivalis $\Delta p o t$ strain and further suppressed in a $\Delta p o t-\Delta o p t$ double-deficient strain. In addition, the growth of the $\Delta p o t$ strain was markedly attenuated and the $\Delta p o t-\Delta o p t$ strain scarcely grew, whereas the $\Delta s s t T$ strain grew well almost like wild type. Consequently, these results demonstrate that predominant uptake of dipeptide in $P$. gingivalis is mostly managed by POT. We thus propose that Pot is a potential therapeutic target of periodontal disease and $P$. gingivalis-related systemic diseases.
\end{abstract}

\title{
INTRODUCTION
}

Porphyromonas gingivalis, an asaccharolytic Gram-negative anaerobe, is a keystone pathogen in chronic periodontitis, a highly prevalent type of chronic inflammation of periodontal tissues. This disease is the main cause of tooth loss in adults, which decreases the quality of life especially in the elderly. In addition, a large number of epidemiological and molecular-based studies have shown a keen association of chronic periodontitis and type 2 diabetes mellitus accounted for the involvement of inflammation and bacterial pathogens like lipopolysaccharide (Grossi and Genco 1998; Lalla and Papapanou 2011). We recently reported a more direct commitment of periodontopathic bacteria to the disease in which bacterial dipeptidyl-peptidase (DPP) 4 modulates blood glucose level through the degradation of incretin peptides, glucagon-like peptide 1 (GLP-1) and 
glucose-dependent insulinotropic polypeptide (GIP) (Ohara-Nemoto et al. 2017). Furthermore, P. gingivalis has been recently detected in the brain, suggesting its association with neurodegeneration in Alzheimer's disease patients (Dominy et al. 2019). Therefore, it is important to elucidate the bacterial metabolic process for disease prevention and care of $P$. gingivalis-related systemic as well as oral diseases.

$P$. gingivalis growth is not supported by carbohydrates but by proteinaceous nutrients, and then the bacterium exclusively utilizes amino acids as its carbon and energy sources (Seddon et al. 1988). Previous studies suggested that most proteinaceous nutrients are incorporated as dipeptides into bacterial cells, since the production of end products such as methyl mercaptan and ammonia is significantly accelerated by the addition of dipeptides (Tang-Larsen et al. 1995; Takahashi and Sato 2001). In accord with these findings, $P$. gingivalis characteristically possesses four kinds of periplasmic dipeptidyl-peptidases (DPPs) that liberate dipeptides from the non-modified $\mathrm{N}$-termini of polypeptides, in contrast to a trace of aminopeptidase activity (Nemoto and Ohara-Nemoto 2016b; Ohara-Nemoto et al. 2018). DPP4 preferentially cleaves a peptide bond on the C-terminal side of penultimate Pro from the N-terminus (P1 position) (Kiyama et al. 1998; Banbula et al. 2000) and P1 Ala with a lesser extent (Mentlein, Gallwitz and Schmidt 1993). DPP4 from $P$. gingivalis degrades GLP-1 and GIP same as the mammalian entity, resulting in elevation and prolongation of postprandial hyperglycemia in the mouse model (Ohara-Nemoto et al. 2017). Both DPP5 (Ohara-Nemoto et al. 2014) and DPP7 (Banbula et al. 2001) are preferential for P1 hydrophobic residues, though DPP7 prefers hydrophobic N-terminal (P2-position) residues (Nemoto et al. 2018) and DPP5 has no such preference (Rouf et al. 2013). Finally, DPP11 is specific for P1 acidic residues, Asp and Glu (Ohara-Nemoto et al. 2011). Accompanying with these DPPs, P. gingivalis expresses prolyl tripeptidyl-peptidase A (PTP-A) (Banbula et al. 1999) and acylpeptidyl oligopeptidase (AOP) (Nemoto et al. 2016a) in the periplasmic space, which provide oligopeptides acceptable for DPPs. These characteristic features of dipeptide production and possible utilization let us presume that amino acid nutrients are transported mainly as a dipeptidyl form via a plasma membrane transporter. In the present study, we aimed to identify the transport molecule responsible for dipeptide uptake in $P$. gingivalis. 


\section{METHODS}

\section{Bacterial growth conditions}

$P$. gingivalis ATCC 33277 were grown at $37^{\circ} \mathrm{C}$ anaerobically $\left(80 \% \mathrm{~N}_{2}, 10 \% \mathrm{CO}_{2}, 10 \% \mathrm{H}_{2}\right)$ in Anaerobic Bacterial Culture Medium (ABCM) broth (EIKEN Chemical, Tochigi, Japan) supplemented with $0.5 \mu \mathrm{g} \mathrm{mL} \mathrm{Lf}^{-1}$ menadione without or with $10 \mu \mathrm{g} \mathrm{mL}^{-1}$ of ampicillin and/or erythromycin. To measure bacterial growth, bacterial cells were collected in an early stationary phase and resuspended in the broth to adjust to 0.2 at $\mathrm{A}_{600}$. Cultures in a 96-well plate were monitored by measuring $\mathrm{A}_{600}$. Growth was also measured by spotting $2 \mu \mathrm{L}$ of 10 -time serially diluted bacterial suspensions from a concentration of $10^{7} \mathrm{cfu} 2 \mu \mathrm{L}^{-1}$ on $\mathrm{ABCM}$ agar plates containing $0.5 \mu \mathrm{g}$ $\mathrm{mL}^{-1}$ of menadione and 5\% sheep blood (blood agar plates). E. coli XL-1 blue and Streptococcus anginosus NCTC 10713 were cultured aerobically at $37^{\circ} \mathrm{C}$ in Luria-Bertani (LB) broth and brain heart infusion broth, respectively.

\section{Substrate uptake assay}

Transporter activities were evaluated by a real-time measurement of bacterial metabolic activity using alamarBlue Cell Viability Reagent composed of resazurin (Thermo Fisher Scientific, Waltham, USA), according to previous reports (Shiloh, Ruan and Nathan 1997; Ishiguro et al. 2015) with slight modifications. In brief, bacterial cells precultured to $\mathrm{A}_{600}$ of 0.8 were harvested, washed twice with ice-cold PBS, and then suspended in $50 \mathrm{mM}$ HEPES, $\mathrm{pH} 7.5$, to $\mathrm{A}_{600}$ of 0.3 . The reagent was added to the cell suspensions giving a final concentration of $2.5 \mathrm{mM}$ and incubated at $25^{\circ} \mathrm{C}$ for $5 \mathrm{~min}$. Measurement was started by the addition of an aliquot $(40 \mu \mathrm{L})$ of the bacterial cell suspension to a reaction mixture (final $200 \mu \mathrm{L}$ ) composed of $50 \mathrm{mM}$ HEPES without or with either $10 \mathrm{mM}$ single amino acids, $5 \mathrm{mM}$ dipeptides, $5 \mathrm{mM}$ each of two kinds of amino acids, or equivalent amounts of peptidyl compounds in an OptiPlate-96F (PerkinElmer). Fluorescence intensity with excitation at $550 \mathrm{~nm}$ and emission at $585 \mathrm{~nm}$ was measured every five min after two sec-shaking for 30 min with a DTX 800 Multimode Detector (Beckman Coulter). Fluorescence intensity $(n=4)$ was subtracted by that without a substrate, then the resulting figures represented genuine incorporation and metabolism mediated by each substrate. Amino acids, 
Gly-Gly, Gly-Gln, Gly-Leu, Gly-Glu, polyE750, and polyE1500 were purchased from Sigma-Aldrich. Gly-Pro, Glu-Glu, and GGG were from Peptide Institute (Ibaraki, Japan), acetyl (Ac)-Gly-Leu and GGP from Bachem (Bubendorf, Switzerland), and Gly-Ser from Tokyo Chemical Industry.

\section{Expression of transporter proteins in $E$. coli}

The serine/threonine transporter (SstT) (PGN_1640), putative oligopeptide transporter (Opt) (PGN_1518), and putative $\mathrm{H}^{+}$/peptide symporter (alias proton-dependent oligopeptide transporter, Pot) (PGN_0135) genes were amplified by PCR with genomic DNA and an appropriate set of primers (Supplementary Table S1). PCR products were cloned into pTrcHis2-TOPO and the constructs were verified by sequencing. E. coli XL1-Blue harboring the plasmid was cultured in LB broth supplemented with $75 \mu \mathrm{g} \mathrm{mL}-1$ of ampicillin at $37^{\circ} \mathrm{C}$ for $16 \mathrm{~h}$, then diluted 3-fold with LB broth and the culture was continued. After 1-h culture, bacterial cells were divided into two aliquots with or without $0.2 \mathrm{mM} \mathrm{IPTG}$, and then further cultured at $30^{\circ} \mathrm{C}$. After $3 \mathrm{~h}$, bacterial cells were harvested and washed with ice-cold PBS and then subjected to further analyses.

\section{Construction of $P$. gingivalis strains disrupting transporter genes}

P. gingivalis strains with disrupted transporter genes were constructed by homologous recombination (Table 1). In short, to construct NDP700 $\Delta s s t T$ (sst T::cepA), DNA fragments from both the 5'- and 3'-parts of the PGN_1640 gene were PCR amplified with primers (Supplementary Table S1). A cepA fragment flanked with parts of PGN_1640 was amplified with primers using pCR4-TOPO as a template. Integrated PCR was performed with a mixture of the three fragments with a set of primers (PGN1640-5F1 and 3R-comp-2PGN1640), then an obtained DNA fragment was introduced into $P$. gingivalis by electroporation. Genedisrupted strains were selected on blood agar plates containing $10 \mu \mathrm{g} \mathrm{mL} L^{-1}$ of ampicillin. Similarly, NDP800 $\Delta o p t(o p t:: c e p A)$ and NDP900 $\Delta p o t$ (pot::cepA) were generated. For the second gene disruption, DNA fragments

of the opt and pot genes were inserted with an erythromycin-resistant gene cassette (ermF ermAM) derived from pYKP301 by integrated PCR, and then the obtained fragments were introduced into the single-gene deficient 
mutants. Double transporter-gene disrupted strains were selected in the presence of $10 \mu \mathrm{g} \mathrm{mL}^{-1}$ each of ampicillin and erythromycin.

\section{Immunoblot analysis}

E. coli cells $(1 \mathrm{~mL})$ were harvested and lysed in SDS-sample buffer $(0.1 \mathrm{~mL})$ containing $5 \mathrm{mM}$ dithiothreitol and $10 \mu \mathrm{L}$ of GLASSMILK suspension (MP Biomedicals) to remove genomic DNA. For $P$. gingivalis cells, $50 \mu \mathrm{M}$ tosyl-L-lysyl-chloromethane hydrochloride, $3 \mu \mathrm{M} \mathrm{E64,} \mathrm{and} 10 \mu \mathrm{g} \mathrm{mL} \mathrm{m}^{-1}$ of leupeptin were further added to inhibit protease activities. After heating and centrifugation, proteins were separated by SDS-PAGE and transferred to a polyvinylidene difluoride membrane. The membrane was incubated with $1 \%$ skim milk in PBS containing $0.05 \%$ Tween 20 and $0.05 \%$ Triton X-100. P. gingivalis transporters were detected using mouse antihexahistidine Ig followed by alkaline phosphatase-conjugated anti-mouse IgG, and visualized with 5-bromo-4chloro-3-indolyl phosphate and nitro blue tetrazolium (Promega). A rainbow marker and low molecular weight markers were purchased from GE Healthcare. Anti- $P$. gingivalis Pot Ig was produced against a synthesized peptide $\left(\mathrm{CM}^{134} \mathrm{YDNDTYRDKR}^{144}\right)$ using a Wistar rat. Non-endogenous ' $\mathrm{C}$ ' was added to facilitate conjugation to a hapten scaffold. After blocking, the membrane was incubated with anti-Pot Ig followed with horseradish peroxidase-conjugated anti-rat IgG, and then the bands were visualized with Western BLoT Ultra Sensitive HPR Substrate (Takara Bio, Kusatsu, Japan). Protein concentration was determined with the Coomassie Brilliant Blue dye method (Promega) using bovine albumin as the standard.

\section{Amino acid sequence alignment and 3D homology modeling}

Amino acid sequences of bacterial POT members were aligned using Clustal W (Larkin et al. 2007). A pyrogenetic tree was created by PhyML of the SeaView multiplatform with the default setting (Gouy, Guindon and Gascuel 2010). Homology modeling of the 3D structure was generated by Phyre2 (Kelley et al. 2015) and visualized using the PyMOL Molecular Graphics System, Ver 2.0 (Schrödinger). 


\section{RESULTS AND DISCUSSION}

\section{Dipeptide incorporation in $P$. gingivalis}

P. gingivalis holds a series of DPPs together with their supporting exopeptidases, PTP-A and AOP, while aminopeptidase activities are scant. These specialized features for producing dipeptides seem to be beneficial for the bacterium to acquire its niche in the oral cavity (Nemoto and Ohara-Nemoto, 2020). Since information on amino acid incorporation of $P$. gingivalis is limited, we firstly examined single amino acid uptake in $P$. gingivalis by real-time measurement of the metabolic activity (Shiloh, Ruan and Nathan 1997; Ishiguro et al. 2015). The higher increase in fluorescence intensity was obtained with Gly, Leu, Ser (Fig. 1), and Thr (data not shown) than Gln, Pro, Glu (Fig. 1), and other amino acids (data not shown). Because Gly provided a high fluorescence intensity and six Gly-Xaa dipeptides and GGG were commercially available, we subsequently used these glycyl compounds. In addition, Glu-Glu and Glu oligopeptides (polyE750 and polyE1500), which have been previously used for the bacterial metabolic study (Takahashi and Sato, 2001), were also tested.

As shown in Fig. 1A, fluorescence intensity increased linearly for $30 \mathrm{~min}$, indicating that substrates incorporated into the bacterial cells were readily metabolized to produce reducing molecules including NADH. The increase was significant in the presence of Gly-Gly and Gly-Leu as compared to those with equivalent amounts of Gly, Leu, and a mixture of Gly and Leu. In contrast, Strep. anginosus and E. coli, saccharolytic bacteria that possess no DPPs, did not show obvious differences in the intensity among the substrates at $30 \mathrm{~min}$ (Fig. 1B). These results indicate that the increase in fluorescence intensity primarily reflects the incorporation efficiency of substrates as compared with the metabolic efficiency of each amino acid at least in $P$. gingivalis. Substrate preference of $P$. gingivalis was further examined (Fig. 1C). The results again demonstrated that $P$. gingivalis more preferentially incorporates dipeptides, particularly Gly-Gly, Gly-Ser, Gly-Gln, and Gly-Leu, while uptake of dipeptides composed of Pro and Glu seemed limited. The efficiency of incorporation of single amino acids (Gly, Ser, Leu), a combination of the two amino acids, and tripeptides GGG and GGP was 15 - 40\% of that of Gly-Gly. The uptake of Gln, Glu, and Pro was limited. Although Gly-Leu was favorable, Ac-Gly-Leu was poorly incorporated (5\% of Gly-Leu), uptake of Poly-Glu750 [(Glu $)_{6}$ as a major part $]$ was scarce, and polyGlu1500 [(Glu $\left.)_{11-20}\right]$ was not incorporated. Taken together, these results confirmed that $P$. gingivalis more 
preferentially incorporates proteinaceous nutrients as dipeptidyl forms than single amino acids, tripeptides and longer oligopeptides.

\section{Functional expression of $P$. gingivalis transporters in $E$. coli}

P. gingivalis ATCC 33277 (Pg) possesses three candidates of amino acid transporter genes (Naito et al 2008):

the serine/threonine transporter sstT (PGN_1640) encoding 412 amino acids with a predicted molecular mass of 41,951 (Dashper et al. 2001), the putative oligopeptide transporter opt (PGN_1518) encoding 659 amino acid residues with a molecular mass of 69,743 , and the putative $\mathrm{H}^{+}$/peptide symporter (alias proton-dependent oligopeptide transporter) pot (PGN_0135) encoding 513 amino acids with a predicted molecular mass of 56,727. To elucidate their roles in substrate uptake, full-length of the three genes were expressed in E. coli as C-terminal hexahistidine tagged molecules (Table 1, Fig. 2). After induction, PgSstT was detected as a 28-kDa band, with 58- and 94-kDa and even larger bands. A small amount of 28-kDa SstT was observed without induction (Fig. 2A, lanes 1 and 2). PgOpt was expressed as a 46-kDa monomer with a 110-kDa dimer, and PgPot as 35-, 76- and $150-\mathrm{kDa}$ bands. These findings suggest a polymerization tendency of the $P$. gingivalis transporters even under denaturing conditions.

Substrate uptake of E. coli expressing P. gingivalis transporters was examined with Ser, Gly-Gly, and GGG, since Ser is a major substrate for SstT (Dashper et al. 2001) and Gly-Gly and GGG recorded maximal incorporation in dipeptidyl and tripeptidyl forms, respectively (Fig. 1C). Inherent uptakes of E. coli cells without and with IPTG induction were subtracted from those values of the cells harboring expressing plasmids, resulting in the figures educed by expression of $P$. gingivalis transporters. The uptake of Ser was significantly enhanced in E. coli expressing PgSstT, whereas those were negligible in both E. coli with PgOpt and PgPot (Fig. 2B).

Incorporation of Gly-Gly was markedly increased in E. coli expressing PgPot after induction, while GGG uptake was enhanced in E. coli-PgOpt. A small GGG uptake observed in E. coli-PgPot was independent of induction. These results suggest that $P$. gingivalis transporters were functionally expressed in E. coli. As a result, it was concluded that Pot is predominantly responsible for dipeptide uptake, Opt for tripeptides, and SstT for amino acids in $P$. gingivalis. 


\section{Characteristics of $\boldsymbol{P}$. gingivalis mutants with disrupted transporter genes}

Single ( $\Delta s s t T, \Delta o p t, \Delta p o t)$ - and double ( $\Delta s s t T-\Delta o p t, \Delta s s t T-\Delta p o t, \Delta o p t-\Delta p o t, \Delta p o t-\Delta o p t)$-transporter genedisrupted $P$. gingivalis strains were constructed (Table 1). Mutant strains were selected by antimicrobial resistances and gene disruption was further confirmed by PCR. The length of inserted cep $A$ gene was $1,117 \mathrm{bp}$ and that of ermF-ermAM was 2,179 bp, and then the amplified sst $T:$ cep $A$ fragment (1,925 bp) was observed in the $\Delta s s t T, \Delta s s t T-\Delta o p t$, and $\Delta s s t T-\Delta p o t$ strains (Fig. 3A, lanes 2, 5, and 6, respectively). The opt::cepA fragment (2,643 bp) was demonstrated in the $\Delta o p t$ and $\Delta o p t-\Delta p o t$ (lanes 3 and 7, respectively), that of the opt::ermF$\operatorname{ermAM}(3,705 \mathrm{bp})$ was in the $\Delta s s t T-\Delta o p t$ and $\Delta p o t-\Delta o p t$ (lanes 5 and 8, respectively), that of the pot::cepA $(2,458$ bp) was present in the $\Delta p o t$ and $\Delta p o t-\Delta o p t$ (lanes 4 and 8, respectively), and the pot::ermF-ermAM (3,520 bp) was in the $\Delta s s t T-\Delta p o t$ and $\Delta o p t-\Delta p o t$ strains (lanes 6 and 7, respectively). Since NDP901 ( $\Delta p o t-\Delta o p t$ ) exhibited a similar growth profile and substrate uptake tendency to those of NDP801 ( $\Delta$ opt- $\Delta p o t)$, the results from NDP801 are solely presented following.

P. gingivalis Pot was shown as the main $35-\mathrm{kDa}$ band together with minor $76-$ and $150-\mathrm{kDa}$ bands, the same as those of Pot molecules expressed in E. coli (Figs. 3B). Although all bands disappeared in the pot mutants, those amounts seemed to decrease in the $\Delta s s t T-\Delta o p t$ strain due to an unknown reason at present. The apparent molecular size of $35 \mathrm{kDa}$ of Pot was smaller than the calculated value (molecular mass $=56,746)$ on SDS-PAGE, and this feature of membrane proteins has been commonly reported, such as E. coli Pot (Weitz et al. 2007).

Uptakes of five Gly-Xaa dipeptides and Glu-Glu were examined using the transporter-deficient strains (Fig. 3C). The $\Delta s s t T$ mutant showed a profile similar to that of the wild type, confirming that SstT does not incorporate dipeptides. In contrast, dipeptide uptakes were significantly reduced in the $\Delta p o t$ strain (approx. $30-$ $60 \%$ each of that in the wild type). Since the $\Delta p o t$ strain maintained certain dipeptide uptake, other molecules such as Opt might function as a dipeptide transporter under these conditions. Unexpectedly, an enhanced dipeptide uptake $(130 \%)$ was observed in the $\Delta o p t$ strain. These findings further suggested that the defect of $o p t$ is likely compensated by the expression of other molecules such as Pot, because of its involvement as an 
adequate role in nutrient uptake. Among the double-deletion mutants, dipeptide uptake was not significantly altered in $\Delta s s t T-\Delta o p t$, and the $\Delta s s t T-\Delta p o t$ strain also exhibited incorporation of dipeptides, except for a significant decrease in Glu-Glu. In contrast, dipeptide uptake was markedly reduced in the $\Delta o p t-\Delta p o t$ strain. These observations again suggest that the defect of either opt or pot gene could be compensated by upregulation of another gene, while such compensation hardly occurred in the $\Delta$ opt- $\Delta p o t$ double-deficient mutant.

The growth of the $P$. gingivalis transporter-deficient strains was investigated in liquid and on blood agar plate cultures. As shown in Fig. 3D, the $\Delta s s t T$ strain showed the least extent of growth retardation, further suggesting that uptake of single amino acids via Sst T is rather limited and does not significantly contribute to bacterial metabolism and energy production. On the other hand, modest growth retardation was observed in the $\Delta o p t$ strain, while that was markedly attenuated in the $\Delta p o t$ strain with a prolonged lag phase. With blood agar plate culture (Fig. 3E), the weakness of the growth of the $\Delta p o t$ strain was reproduced. Furthermore, the growth of the $\Delta s s t T-\Delta o p t$ was more weakened than those of their single mutants, and the $\Delta o p t-\Delta p o t$ strain failed to grow except with the highest level of inoculation. These results indicate that dipeptide uptake mainly managed by Pot is most closely associated with bacterial growth and suggest that Opt appears to have a subsidiary role in substrate incorporation. SstT seemed to have the least role in the growth under these conditions.

\section{Amino acid sequence comparison and 3D structure modeling of $P$. gingivalis Pot}

The amino acid sequence of $P$. gingivalis Pot (PgPot) exhibits 29.6\% similarity with that of Geobacillus kaustophilus Pot (GkPOT), followed by $29.2 \%$ to that of Shewanella oneidensis $\mathrm{Pep}_{\mathrm{So}}, 27.7 \%$ to that of E. coli dipeptide and tripeptide permease B (DtpB), and 27.3\% to that of Streptococcus thermophilus Pep $_{\mathrm{St}}$, while the phylogenetic tree shows a closer kinship with E. coli DtpB than S. oneidensis $\operatorname{PepT}_{\mathrm{So}}$ (Supplemental Fig. S1). The amino acid sequence alignment with other bacterial entities, in which 3D structures have been determined, indicates that identical amino acid residues are mainly located at the N-terminal half (Fig. 4). The conserved 'ExxER/K' motif in the POT members was shown to change to ' $\mathrm{N}^{16} \mathrm{MGER}^{20}$ ' in PgPot, though Glu ${ }^{32}$ in GkPot has been reported to be essential for proton-driven uptake of substrates (Doki et al. 2013). KEGG orthologue search revealed that the 'NxGER' sequence is conserved in the members in the order Bacteroidales including 
periodontopathic bacteria such as Tannerella forsythia and Prevotella intermedia, and gut indigenous bacteria such as Bacteroides fragilis, Bacteroides thetaiotaomicron, and Alistipes onderdonkii, as well as the species in the family Porphyromonadaceae. Because of the medical importance, it is interesting to examine whether amino acid substitutions in the conserved motif provides specific characteristics to Bacteroidales POT members.

Homology modeling of the 3D structure (471 of 513 residues, $92 \%$ ) was generated with $100 \%$ confidence by the single highest scoring template of GkPot (Doki et al. 2013), showing that PgPot consists of the conserved N-bundle transmembrane regions (TM) 1-6 and C-bundle TM 7-12, with the $\alpha$ helix regions HA and HB (Fig. 5). The N- and C-bundles surround and form a large central cleft, thus providing a substrate-binding site. Since diverse substrate promiscuity is recognized as a characteristic feature of the POT members (Weitz et al. 2007; Ito et al. 2013; Newstead 2015), it is of interest to elucidate that the 3D structure and the substrate multispecificity of P. gingivalis Pot.

\section{CONCLUSIONS}

P. gingivalis preferentially incorporates dipeptides as nutritional amino acids, which is managed predominantly by Pot. The other two transporters, i.e., Opt mainly responsible for tripeptides and SstT for single amino acids, play subsidiary roles in uptakes of nutritional amino acids. Bacterial growth was significantly retarded in the $\Delta p o t$ mutant, and the $\Delta p o t-\Delta o p t$ double mutant scarcely grew under the present culture conditions. Thus, dipeptide production by DPPs and their uptake by Pot are conclusive molecular events in P. gingivalis for the entire metabolism and energy production. The present results indicate that the Pot molecule can be a potential drug target for the prevention and care of periodontal disease as well as $P$. gingivalis-related systemic diseases such as type 2 diabetes mellitus and Alzheimer's disease.

\section{SUPPLEMENTARY DATA}

Supplementary data are available at FEMS online. 


\section{ACKNOWLEDGMENTS}

We are grateful for the technical assistance of Dr. T.T. Baba and Dr. T. Ono (Nagasaki University).

\section{FUNDING}

This study was supported by JSPS KAKENHI Grant Numbers 18K09557 (to Y.S.), 19K10045 (to T.K.N.), 19K10071 (to Y.O.-N.) and a grant from the Kyushu Dentist Association (to T.K.N.).

Conflicts of Interest. None declared.

\section{REFERENCES}

Banbula A, Mak P, Bugno M et al. Prolyl tripeptidyl peptidase from Porphyromonas gingivalis. A novel enzyme with possible pathological implications for the development of periodontitis. J Biol Chem 1999;274:924652.

Banbula A, Bugno M, Goldstein J et al. Emerging family of proline-specific peptidases of Porphyromonas gingivalis: purification and characterization of serine dipeptidyl peptidase, a structural and functional homologue of mammalian prolyl dipeptidyl peptidase IV. Infect Immun 2000;68:1176-82.

Banbula A, Yen J, Oleksy A et al. Porphyromonas gingivalis DPP-7 represents a novel type of dipeptidylpeptidase. J Biol Chem 2001;276:6299-305.

Dashper SG, Brownfield L, Slakeski N et al. Sodium ion-driven serine/threonine transport in Porphyromonas gingivalis. J Bacteriol 2001;183:4142-8.

Doki S, Kato HE, Solcan N et al. Structural basis for dynamic mechanism of proton-coupled symport by the peptide transporter POT. Proc Natl Acad Sci USA 2013;110:11343-8.

Dominy SS, Lynch C, Ermini F et al. Porphyromonas gingivalis in Alzheimer's disease brains: Evidence for disease causation and treatment with small-molecule inhibitors. Sci Adv 2019:23:eaau3333. 
Gouy M, Guindon S, Gascuel O. SeaView version 4: A multiplatform graphical user interface for sequence alignment and phylogenetic tree building. Mol Biol Evol 2010;27:221-4.

Grossi SG, Genco RJ. Periodontal disease and diabetes mellitus: a two-way relationship. Ann Periodonto 1998;3:51-61.

Ishiguro K, Washio J, Sasaki K et al. Real-time monitoring of the metabolic activity of periodontopathic bacteria. J Microbiol Methods 2015;115:22-6.

Ito K, Hikida A, Kawai S, et al. Analysing the substrate multispecificity of a proton-coupled oligopeptide transporter using a dipeptide library. Nat Commun 2013;4:2502 doi: 10.1038/ncomms3502.

Kelley LA, Mezulis S, Yates CM et al. The Phyre2 web portal for protein modeling, prediction and analysis. Nat Protoc 2015;10:845-58.

Kiyama M, Hayakawa M, Shiroza T et al. Sequence analysis of the Porphyromonas gingivalis dipeptidyl peptidase IV gene. Biochim Biophys Acta 1998;1396:39-46.

Lalla E, Papapanou PN. Diabetes mellitus and periodontitis: a tale of two common interrelated diseases. Nat Rev Endocrinol 2011;7:738-48.

Larkin MA, Blackshields G, Brown NP et al. Clustal W and Clustal X version 2.0. Bioinformatics 2007;23:2947-8.

Mentlein R, Gallwitz B, Schmidt WE. Dipeptidyl-peptidase IV hydrolyses gastric inhibitory polypeptide, glucagon-like peptide-1(7-36)amide, peptide histidine methionine and is responsible for their degradation in human serum. Eur J Biochem 1993;214:829-35.

Naito M, Hirakawa H, Yamashita A et al. Determination of the genome sequence of Porphyromonas gingivalis strain ATCC 33277 and genomic comparison with strain W83 revealed extensive genome rearrangements in P. gingivalis. DNA Res 2008;15:215-25.

Nemoto TK, Ohara-Nemoto Y, Bezerra GA et al. A Porphyromonas gingivalis periplasmic novel exopeptidase, acylpeptidyl oligopeptidase, releases $\mathrm{N}$-acylated di- and tripeptides from oligopeptides. $J$ Biol Chem 2016a;291:5913-25.

Nemoto TK, Ohara-Nemoto Y. Exopeptidases and gingipains in Porphyromonas gingivalis as prerequisites for its amino acid metabolism. Jpn Dent Sci Rev 2016b;52:22-9. 
Nemoto TK, Ono T, Ohara-Nemoto Y. Establishment of potent and specific synthetic substrate for dipeptidylpeptidase 7. Anal Biochem 2018;548:78-81.

Nemoto TK, Ohara-Nemoto Y. Dipeptidyl-peptidases: Key enzymes producing entry forms of extracellular proteins in asaccharolytic periodontopathic bacterium Porphyromonas gingivalis. Mol Oral Microbiol 2020; doi: 10.1111/omi.12317.

Newstead S. Molecular insights into proton coupled peptide transport in the PTR family of oligopeptide transporters. Biochim Biophys Acta 2015;1850:488-99.

Ohara-Nemoto Y, Shimoyama Y, Kimura S et al. Asp- and Glu-specific novel dipeptidyl peptidase 11 of Porphyromonas gingivalis ensures utilization of proteinaceous energy sources. $J$ Biol Chem 2011;286:38115-27.

Ohara-Nemoto Y, Rouf SM, Naito M et al. Identification and characterization of prokaryotic dipeptidylpeptidase 5 from Porphyromonas gingivalis. J Biol Chem 2014;289:5436-48.

Ohara-Nemoto Y, Nakasato M, Shimoyama Y et al. Degradation of incretins and modulation of blood glucose levels by periodontopathic bacterial dipeptidyl peptidase 4. Infect Immun 2017;85(9):pii: e00277-17.

Ohara-Nemoto Y, Shimoyama Y, Nakasato M et al. Distribution of dipeptidyl peptidase (DPP) 4, DPP5, DPP7 and DPP11 in human oral microbiota—potent biomarkers indicating presence of periodontopathic bacteria. FEMS Microbiol Lett .2018;365:doi: 10.1093/femsle/fny221.

Rouf SM, Ohara-Nemoto Y, Ono T et al. Phenylalanine 664 of dipeptidyl peptidase (DPP) 7 and phenylalanine 671 of DPP11 mediate preference for P2-position hydrophobic residues of a substrate. FEBS Open Bio 2013;3:177-83.

Seddon SV, Shah HN, Hardie JM et al. Chemically defined and minimal media for Bacteroides gingivalis. Curr Microbiol 1988;17:147-9.

Shiloh MU, Ruan J, Nathan C. Evaluation of bacterial survival and phagocyte function with a fluorescence-based microplate assay. Infect Immun 1997;65:3193-8.

Takahashi N, Sato T. Preferential utilization of dipeptides by Porphyromonas gingivalis. J Dent Res 2001;80:1425-9.

Tang-Larsen J, Claesson R, Edlund MB et al. Competition for peptides and amino acids among periodontal bacteria. J Periodontal Res 1995;30:390-5. 
Weitz D, Harder D, Casagrande F et al. Functional and structural characterization of a prokaryotic peptide transporter with features similar to mammalian PEPT1. J Biol Chem 2007;282:2832-9. 
Table 1. Bacterial strains used in this study.

\begin{tabular}{|c|c|c|c|}
\hline Bacteria & Strain & Genotype & Source or reference \\
\hline \multirow[t]{8}{*}{$P$. gingivalis } & ATCC 33277 & - & ATCC \\
\hline & NDP700 & sstT::cepA & This study \\
\hline & NDP701 & sst $T::$ cepA opt::[ermF ermAM] & This study \\
\hline & NDP702 & sst $T::$ cepA pot::[ermF ermAM] & This study \\
\hline & NDP800 & opt::cepA & This study \\
\hline & NDP801 & opt::cepA pot::[ermF ermAM] & This study \\
\hline & NDP900 & pot::cepA & This study \\
\hline & NDP901 & pot::cepA opt::[ermF ermAM] & This study \\
\hline E. coli & XL-1 Blue & - & - \\
\hline Strep. anginosus & NCTC 10713 & - & NCTC \\
\hline
\end{tabular}


A

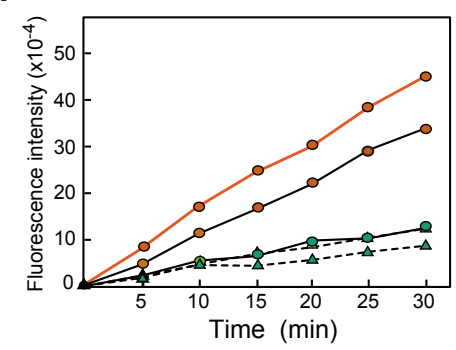

C

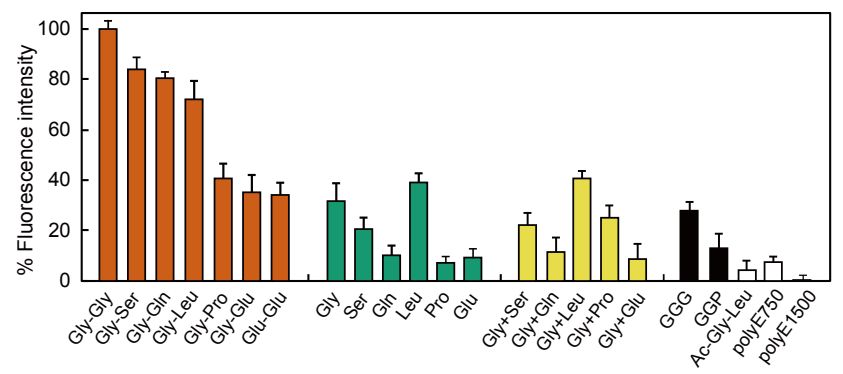

Dipeptide, amino acid, or polypeptide
B

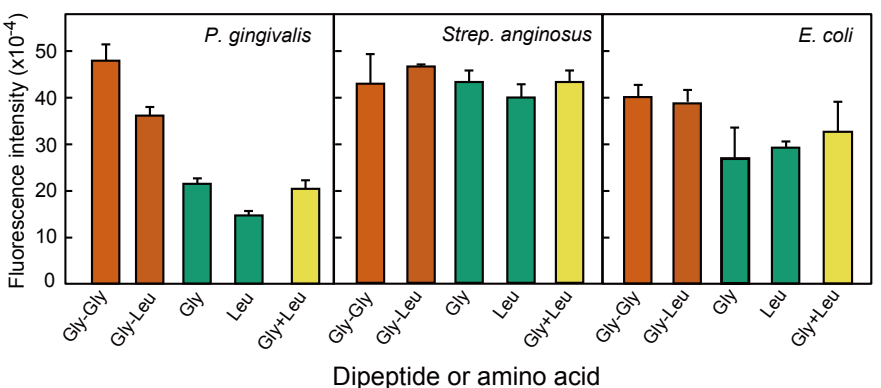

Dipeptide or amino acid

Figure 1. Substrate uptake potential determined by real-time metabolic activity.

(A) Fluorescence intensity was measured with $P$. gingivalis pre-incubated with resazurin reagent with either 5 mM Gly-Gly or Gly-Leu, $10 \mathrm{mM}$ Gly or Leu, or $5 \mathrm{mM}$ each of Gly and Leu. The average was shown. (B) Substrate uptake was measured and fluorescence intensity at $30 \mathrm{~min}$ is depicted for $P$. gingivalis, Strep. anginosus, and E. coli. (C) The analysis with $P$. gingivalis was performed with at identical moles as amino acids (5 mM Gly-Xaa and Glu-Glu, $10 \mathrm{mM}$ single amino acid, $5 \mathrm{mM}$ of each combination of amino acids, $3.3 \mathrm{mM}$ GGG, GGP, $5 \mathrm{mM}$ Ac-Gly-Leu, $1.7 \mathrm{mM}$ poly E750, $0.7 \mathrm{mM}$ polyE1500). Representative results at $30 \mathrm{~min}$ are shown as the average \pm S.D. $(n=4)$, and four independent measurements were performed. 
A

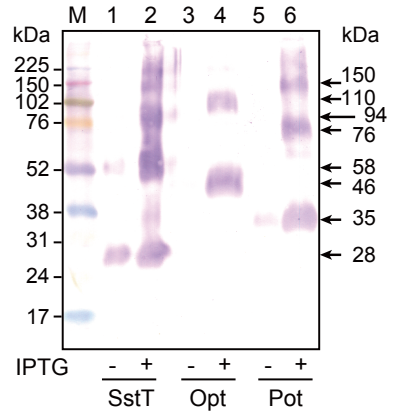

B

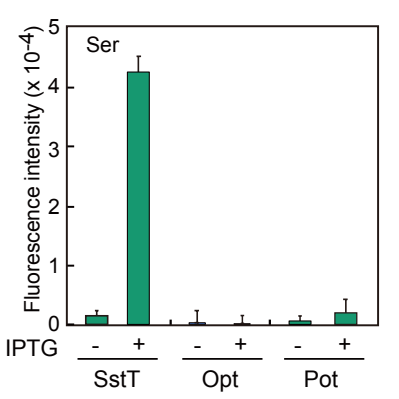

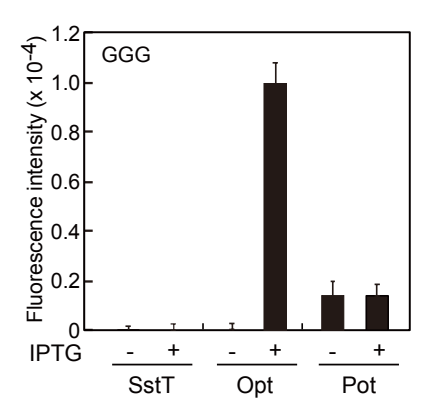

Figure 2. Expression of $P$. gingivalis transporters in E. coli.

(A) Western blot analysis of $P$. gingivalis SstT, Opt , and Pot expressed in E. coli with pTrcHis2-TOPO-sstT-, $o p t$, and pot, respectively. Whole bacterial cell lysates were subjected to immunoblotting with an antihexahistidine Ig. (B) E. coli cells were cultured with or without IPTG. After washing and preparation of bacterial cell suspensions, substrate uptake was measured with $10 \mathrm{mM}$ Ser, $5 \mathrm{mM}$ Gly-Gly, and $3.3 \mathrm{mM}$ GGG for $30 \mathrm{~min}$. Values were obtained by subtraction of endogenous uptake of $E$. coli without plasmids. Representative results from three independent experiments are shown as the average \pm S.D. $(n=4)$. 
A

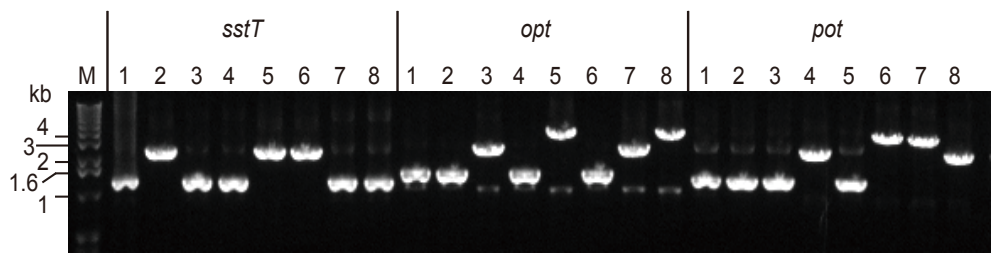

C

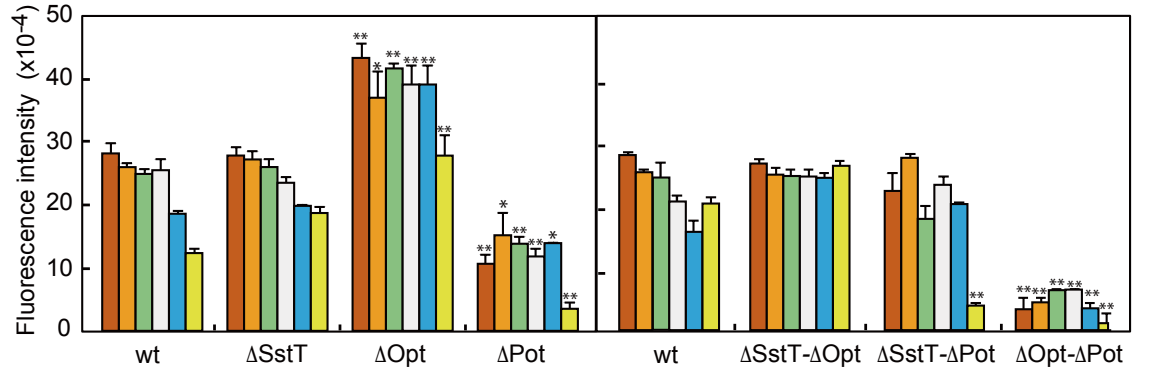

$\mathrm{D}$

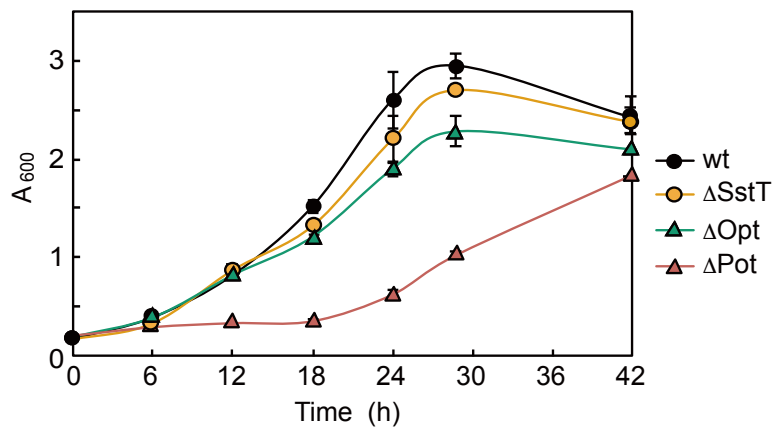

B

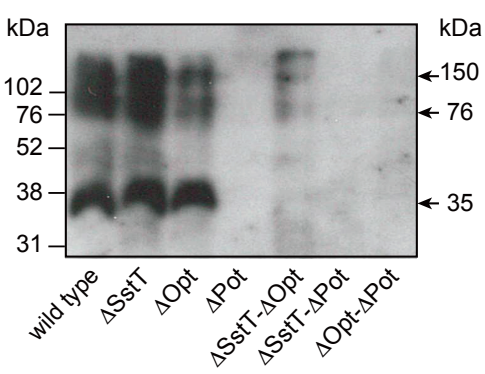

$\square$ Gly-Gly

$\square$ Gly-Leu

$\square$ Gly-Ser

$\square$ Gly-Phe

$\square$ Gly-Glu

口Glu-Glu

E

\begin{tabular}{|c|c|c|c|c|c|}
\hline & $x 1$ & $x 10^{-1}$ & $x 10^{-2}$ & $\times 10^{-3}$ & $\times 10^{-4}$ \\
\hline wt & (2) & (a) & (2) & e: & 8 \\
\hline$\Delta$ SstT & (1) & 0 & 0 & . & 9 \\
\hline$\Delta \mathrm{Opt}$ & 0 & ( & (2) & is & $\circ$ \\
\hline$\Delta$ Pot & (C) & ( ) & ○ & & E \\
\hline$\Delta$ SstT- $\Delta$ Opt & (c) & (c) & a & $x$ & . \\
\hline$\Delta$ SstT- $\Delta$ Pot & (c) & (3) & 。 & 。 & $\circ$ \\
\hline$\Delta$ Opt- $\Delta$ Pot & (1) & $\&$ & ? & & $a$ \\
\hline
\end{tabular}

Figure 3. Characteristics of transporter-deficient $P$. gingivalis strains.

(A) Transporter-deficient $P$. gingivalis strains were confirmed by PCR using sets of primers for nested PCR and genomic DNA from wild type (lane 1), $\Delta s s t T$ (lane 2), $\Delta$ opt (lane 3), $\Delta p o t$ (lane 4), $\Delta s s t T-\Delta o p t$ (lane 5), $\Delta s s t T$ $\Delta p o t$ (lane 6), $\Delta o p t-\Delta p o t$ (lane 7), and $\Delta p o t-\Delta o p t$ (lane 8). (B) Whole cell lysates (5 $\mu$ g of protein) were subjected to SDS-PAGE and $P$. gingivalis Pot was detected with ECL immunoblotting. (C) Dipeptide uptakes were measured by the real-time metabolic assay. Representative results at $30 \mathrm{~min}$ are shown as the average \pm S.D. ( $\mathrm{n}=$ 4), and three independent measurements were performed. ${ }^{*} p<0.05,{ }^{*} p<0.01$, as compared with wild-type (Student's $t$-test). (D) P. gingivalis strains were grown in ABCM broth. The average \pm S.D. was calculated $(\mathrm{n}=$ 4). (E) P. gingivalis strains at $10^{7}$ cfu $2 \mu \mathrm{L}^{-1}$ and 10 -fold serially diluted bacterial cell suspensions were inoculated on blood agar plates and cultured for $42 \mathrm{~h}$. 
TM helix 1

PgPot PepTSh SauPepT GkPot PepTSt LlDptT PepTSo EcDtpB

PgPot PepTSh SauPepT GkPot PepTSt LlDptT PepTSo EcDtpB

PgPot PepTSh SauPepT GkPot PepTSt LlDptT PepTSo EcDtpB

PgPot PepTSh SauPepT GkPot PepTSt LlDptT PepTSo EcDtpB

PgPot PepTSh SauPepT GkPot PepTSt LlDptT PepTSo EcDtpB

PgPot PepTSh SauPepT GkPot PepTSt LlDptT PepTSo EcDtpB

PgPot PepTSh SauPepT GkPot PepTSt LlDptT PepTSo EcDtpB

PgPot PepTSh SauPepT GkPot PepTSt LlDptT PepTSo ECDtpB (n) MATNNSHEQTIQSIPQKGFFGHP---RGLGVLFFVEFWERFSYYGMRAMLIFY--MYFAIHQNGLGIDKTTAMSIMS MTQQNSHGNQIQDIPQTGFFGHP---RGLGVLFFVEFWERF SYYGMRALLIFY--MYFAVTDNGLGIDKTTAMSIMS -MASIDKQQIAASVPQRGFFGHP---KGLFTLFFTEFWERF SYYGMRAILVYY--MYYEVSKGGLGLDEHLALAIMS ---MEDKG--------KTFFGQP---LGLSTLFMTEMWERF SYYGMRAILLYY-MWFLISTGDL----H ITRATAAS MONLNKTE--------KTFFGQP---RGLLTLFQTEFWERF SYYGMRAILVYYLYALTTADNAGLGLPKAOAMAIVS --MSVAKPQ-------GTMLGHP---KGLFLLFTTELWERFSYYAMRAILVLYLVDQVGKOGGGLGWTOADALSLYG ----MNT--------TTPMGMLQQPRPFFMIFFVELWERFGYYGVQG-------VLAVFFVKQLGF SQEQAFVTFG

TM helix 2

TMhelix 2

TMhelix 2 IFYASVYVLÅLVG---GI IADSMKNYKGTILVG்LIVMAAGYVMLGIPTPTQATंGMTPWLIFTCंAALAF ISFGN்GLFK 124 VYGALIYMSSIPG---AWIADRITGTRGATLLGAVLIIIGHICL---SLPFALF-------GLFSSMFFIIIGSGLMK 137 VYGSLI YMTS IPG---GWIADRITGTRGATLLGAVFII IGHICL---SLPFALI--_---GLFTSMFFIIIGSGLMK 137 IYGALVYMSGI IG---GWLADRVFGTSRAVFYGGLLIMAGHIAL---AIPGGVA------ALFVSMALIVLGTGLLK 136 IMAIYASMVYLSGTIGGFVADRI IGARPAVFWGGVLIMLGHIVL---ALPFGAS------ALFGSIILIIIGTGFLK 126 IYGALVYLSTIVG---GWVADRLLGASRTIFLGGILITLGHIAL---ATPFGLS------SLFVALFLIILGTGMLK 131 TFTALVYLTPLIG---GWLADNFLGORKAIYFGGALMATGOFML---AAPHAWFPGIETTVFYIGLGTLILGNGLFK 136 AFAALVYGLISIG---GYVGDHLLGTKRTIVLGALVLAIGYFMTG--MSLLKPDLIFIALGTIAV------GNGLFK 123

TMhelix 5

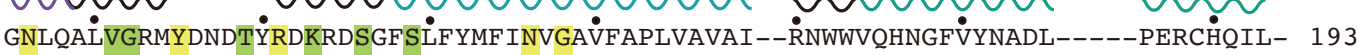
PNISNIVGRLYPENDTRIDAGFVIF--YMSVNLGALISPIILQHF--VDIRNFHGGFLLAAIG-MALGLVWYLL--- 206 PNISNIVGRLYPENDRRMDAGFVIF--YMSVNMGALLSPI ILQHF--VNVKNFHGGFLIAAVG-MALGLVWYVL--- 206 PNVSS IVGDMYKPGDDRRDAGFS IF--YMGINLGAFLAPLVVGTA--GMKYNFHLGFGLAAVG-MFLGLVVFVA--- 205 PNVSTLVGTLYDEHDRRRDAGFSIF--VFGINLGAFIAPLIVGAA--QEAAGYHVAFSLAAIG-MFIGLLVYYF--- 195 PNISNMVGHLYSKDDSRRDTGFNIF--VVGINMGSLIAPLIVGTV--GQGVNYHLGFSLAAIG-MIFALFAYWY--- 200 PNISTMVGDLYEEGDHRRDGAFTIF--YMGINLGAALSGFVVAWAYTSFGHAEVINGKEVFIN-NWQAGFFCAGIGM 210 ANPASLLSKCYPPKDPRLDGAFTLF--YMSINIGSLIALSLAPVIADRFGYSVTYNLCGAGLIIALLVYIACRG--- 195

\section{r}

-NGTLPENAAKAOVMEMIO̊AANNGT--AVATTEGLOEFALKY்IOVF STGFHŸAFLAAVFFMÄI 251 -_--_-_-_NRKNLGSVGMKPTNPLSKEEKRK---YGMIIG--_-_-----IIVAIVIVVLLVTYYTHTL 257 -------------FNRKNLGSVGMKPTNPLTPAEKKK---YGLIIG----------S-VVLAIVLIIVIGALTNS 256 ------------TRKKNLGLAGTYVPNPLTPAEKKK---AAAIMA----------V-GAVVIAVLLAILIPNG- 252 ---_-_---_---_GGKKTLDPHYLRPTDPLAPEEVKPLL-VK---V----------SLAVAGFIAIIVVMNLVGW 243 -------------GRLRHFPEIGREPSNPMDSKARRNFL-ITLTIV----------VIVAIIGFFLLYQASPANF 251 LLSLVIQFLFAQKLLGDIGTVPAARLERERQAKLGNVRKEPLTKVERD-----------RIKVIMVLGLFTIIFWAGF 277

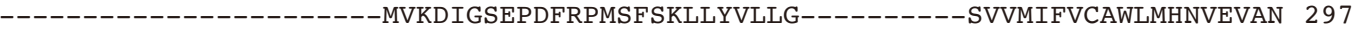

SFLIYIINKH QYPADQKA----------NAVTEAHKDQKOEIKMAADEIRQRIIALCAVFGVVIFFWMSFHQNGVS 317

SFLIYIINKH QYPADQKA----------NAVTEAHKDQKOEIKMAADEIRQRIIALCAVFGVVIFFWMSFHQNGVS 317

TM helix 7 ----SFDLISNTVLVLGV--ALPI IYFTTMLRSKDVTDGE---RSRVK--AF IPLF ILGMLFWS IQEQGSNVLNI-Y 320 L---SFNLVSNTVLVLGI--ALPI IYFTLI IRSKDVTDTE---RSRVK--AF IPLF ILGMVFWAIOEQGSNVLNI-Y 320 ------WFTVETFISLVGILGI I IPI IYFVVMYRSPKTTAEE-RSRVI--AYIPLFVASAMFWAIOEOGSTILAN-Y 319 ----NSLPAYINLLTIVA---IAIPVFYFAWMISSVKVTSTE-HLRVV--SYIPLFIAAVLFWAIEEQGSVVLATFA 310 I---NNF INVLSIIGIVV---PII---YFVMMFTSKKVESDE-RRKLT--AYIPLFLSAIVFWAIEEQSSTIIAV-W 315 EOA-GGLMNLFTNEFTDR---------YIGTWEVPTTYFO---SLNAI--FIVLFAPVVASIWIR--LGKNEPNSPV 337 L--------VLIVLSIVVT------IIFFRQAFKLDKTGRNK-MFVAF--VLMLEAVVFYILYAQMPTSLNFFAI-- 297

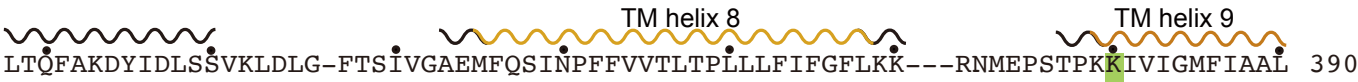

TM helix 9 GLERSDMOLNLFGWTTRFGEALFQS INPLF ILLFAPVISMIW---------LK--MGKKOPSLAIKFSIGTLLAGL 385 GIEHSDMKLNLFGWKTNFGEAIFOS INPLFILLLAPI ISLLW----------OK--LGTKOPSLPVKFAIGTFLAGA 385 A--DKRTQLD--VAGIHLSPAWFQSLNPLF I I ILAPVFAWMW----------VK--LGKRQPTI PQKFALGLLFAGL 380 AERVDSSWFPV--------SWFQSLNPLFIMLYTPFFAWLW----------TA--WKKNQPSSPTKFAVGLMFAGL 366 GESRSNLDPTWFGITFHIDPSWYOLLNPLF IVLLSPIFVRLW----------NK--LGEROPSTIVKFGLGLMLTGI 380 KF-ALGLVLLAIGFLFMIGAVVE---_-_-_--_----MGGD-_---_----AS--AKSS----MWWLVGAYFFHTM 382 ---NNVHHEIL---GFS INPVSFQALNPFWVVLASPILAGIY----------THLGNKGKDLSMPMKFTLGMFMCSL 358

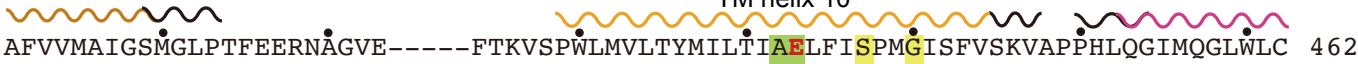

TM helix 10 SYILIGLVGL-------GYGHT------OFSVN--WVILSYVICVIGELCLSPTGNSAAVKLAPKAFNAQMMSVWLL 447 SYILIGIVGY-------ASGSS------NFSVN--WVILSYIICVIGELCLSPTGNSAAVKLAPKAFNAOMMSIWYL 447 SFI--VILVP-------GHLSG------GGLVHPIWLVLSYF IVVLGELCLSPVGLSATTKLAPAAFSAQTMSLWFL 442 SFLLMAIPGA-------LYGTS------G-KVSPLWLVGSWALVILGEMLISPVGLSVTTKLAPKAFNSQMMSMWFL 429 SYLIMTLPGL-------LNGTS------G-RASALWLVLMFAVOMAGELLVSPVGLSVSTKLAPVAFOSOMMAMWFL 450 GELCLSPIGL-------SMVTKLAPLRIASLMMGAWFLFVAAANKIGGVVGSF IGHGGEKEEQLANAMAIFSGIAIT 452 GFLTAAAAGM-------WFADA------QGLTSPWF IVLVYLFQSLGELF ISALGLAMIAALVPQHLMGF ILGMWFL 422 
Figure 4. Sequence alignment of the POT family members and secondary structure of $P$. gingivalis Pot. Amino acid sequences of $P$. gingivalis Pot (PgPot,UniProt: B2RH09), Staphylococcus hominis $\mathrm{PepT}_{\mathrm{Sh}}$ (A0A533J3Z5), Staphylococcus aureus SauPepT (A0A0H2XIN4), Geobacillus kaustophilus GkPot (Q5KYD1), Streptococcus thermophilus $\mathrm{PepT}_{\mathrm{St}}(\mathrm{Q} 5 \mathrm{M} 4 \mathrm{H} 8)$, Lactococcus lactis LiDptT (P0C2U2), Shewanella oneidensis $\mathrm{PepT}_{\mathrm{So}}$ (K4PU14), and E. coli DtpB (P36837) were aligned. Identical residues in 8 and 7 molecules are highlighted in yellow and green, respectively, and essential amino acid residues in the conserved ExxER/K motif and 5 residues involved in $\mathrm{H}^{+}$-driven substrate uptake proposed for GkPot are presented in bold red.

Transmembrane (TM) helices, additional intermediate helix A (HA) and HB, and $\alpha$-helices in black are indicated within the sequences predicted by Phyre2 modeling. 


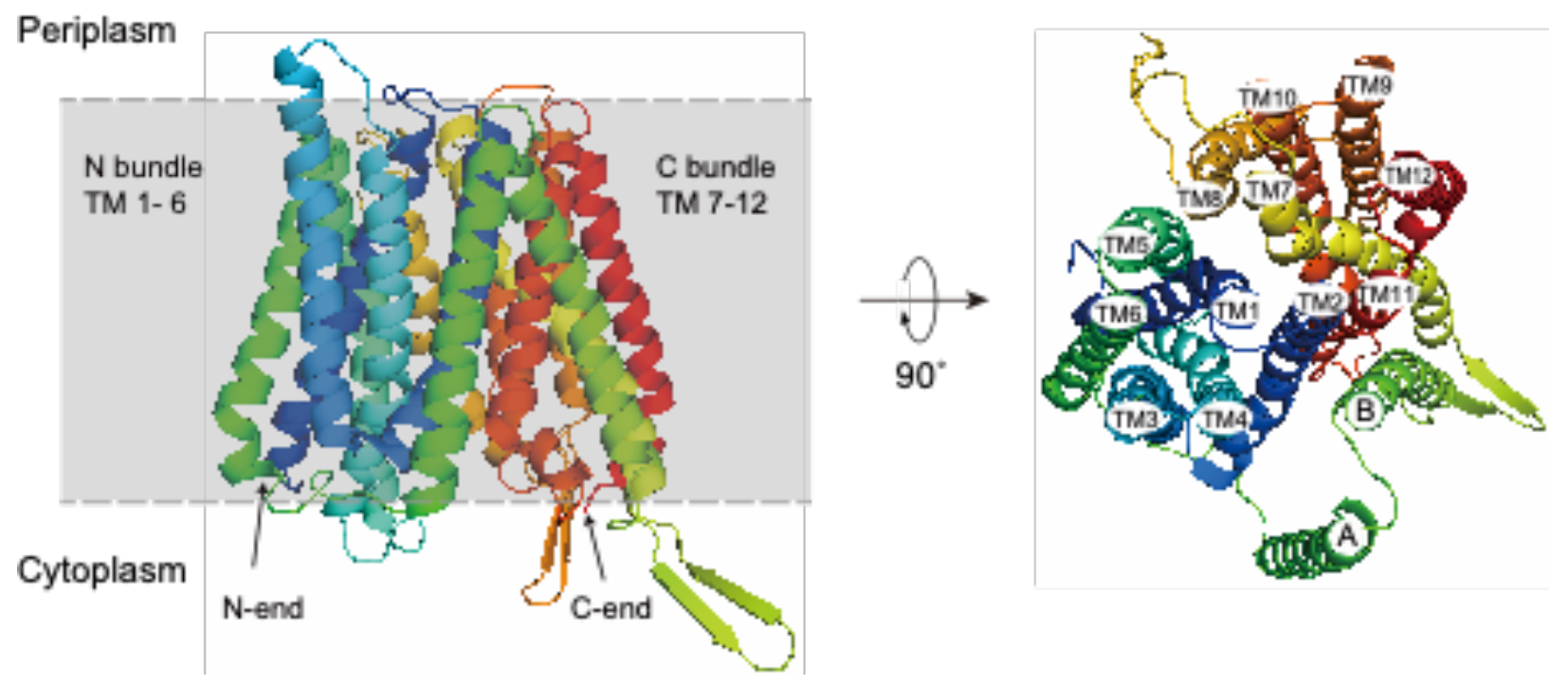

Figure 5. 3D homology modeling of $P$. gingivalis Pot.

A 3D homology model was generated by Phyre2 in the plane of the inner membrane. The canonical $\mathrm{N}$ bundle of TM $1-6$ and C bundle of TM 7 - 12, with additional intermediate helix A (A) and helix B (B), are indicated. 
Supplemental Table S1. Primers used in this study.

\begin{tabular}{|c|c|c|}
\hline Usage & Name & Sequence (5'-3') \\
\hline \multirow[t]{2}{*}{ sstT 5' fragment-cepA } & PGN1640-5F $1 * 1$ & atgcgtaaactacggatcggcctct \\
\hline & PGN1640-5R-cep-comp & TGCTTCACACtggcaatgaatcetgccaccg \\
\hline \multirow[t]{2}{*}{ sstT-cepA } & PGN1640-5F-cepF & gattcattgccaGTGAAGCATCTTCGATGCTG \\
\hline & PGN1640-3R-comp & aaggattcttctCCGATAGTGATAGTGAACGG \\
\hline \multirow[t]{2}{*}{ sstT 3' fragment-cepA } & cep-3F-PGN1640-3F & TCACTATCGGagaagaatcctttcaggatgc \\
\hline & 3R-comp-PGN1640 & cgggcagacatatactgegecatcc \\
\hline \multirow[t]{2}{*}{ sst $T$ nested PCR } & PGN1640-5F2 & ttgcccaagatcatattgg \\
\hline & 3R-comp-2-PGN1640 & agagagggatcgtaaagcc \\
\hline \multirow[t]{2}{*}{ opt 5 ' fragment-cep $A$} & PGN1518-5F1*2 & tcatcggcggttgtctcggtatatt \\
\hline & PGN1518-5R-cep-comp & TGCTTCACACaaaagcatcgttaccaccaaa \\
\hline \multirow[t]{2}{*}{ opt-cepA } & PGN1518-5F-cepF & aacgatgctttGTGAAGCATCTTCGATGCTG \\
\hline & PGN1518-3R-comp & ttgaattggaagCCGATAGTGATAGTGAACGG \\
\hline \multirow[t]{2}{*}{ opt 3' fragment-cep $A$} & cep-3F-PGN1518-3F & TCACTATCGGcttccaattcaacgtgctgtt \\
\hline & 3R-comp-PGN1518*3 & ttatgcttcatgtttcttgcetctg \\
\hline \multirow[t]{2}{*}{ opt nested PCR } & PGN1518-5F2 & tgctattgattcctttccg \\
\hline & 3R-comp-2-PGN1518 & gtcgacgatgaagtagagaat \\
\hline \multirow[t]{2}{*}{ opt 5' fragment-erm } & PGN1518-5F1*2 & tcatcggcggttgtctcggtatatt \\
\hline & PGN1518-5R-erm-comp & CAATAGCGGAAGCTaaaagcatcgttaccaccaaa \\
\hline \multirow[t]{2}{*}{ opt-erm } & PGN1518-5F-ermF & aacgatgcttttAGCTTCCGCTATTGCTTTTTTGC \\
\hline & PGN1518-3R-comp-erm & ttgaattggaagCTCTAGAGGATCCCCGAAGCTG \\
\hline \multirow[t]{2}{*}{ opt 3' fragment-erm } & erm-3F-PGN1518-3F & GATCCTCTAGAGcttccaattcaacgtgctgtt \\
\hline & 3R-comp-PGN1518*3 & ttatgcttcatgtttettgcetctg \\
\hline \multirow[t]{2}{*}{ pot 5' fragment-cep $A$} & PGN0135-5F $1 * 4$ & atcttctatgcttcggtatatgtattg \\
\hline & PGN0135-5R-cep-comp & TGCTTCACACaactgcgcaaagagcaataat \\
\hline \multirow[t]{2}{*}{ pot-cepA } & PGN0135-5F-cepF & ctttgcgcagttGTGAAGCATCTTCGATGCTG \\
\hline & PGN0135-3R-comp & aacgacaccgaaCCGATAGTGATAGTGAACGG \\
\hline \multirow[t]{2}{*}{ pot 3' fragment-cepA } & cep-3F-PGN0135-3F & TCACTATCGGttcggtgtcgttattttcttc \\
\hline & 3R-comp-PGN0135*5 & ttacttcgctaccegctccagccac \\
\hline \multirow[t]{2}{*}{ pot nested PCR } & PGN0135-5F2 & tgggctctcgtgggaggta \\
\hline & 3R-2-PGN0315 & aaccatggagagcataacgag \\
\hline \multirow[t]{2}{*}{ pot 5' fragment-erm } & PGN0135-5F $1 * 4$ & atcttctatgcttcggtatatgtattg \\
\hline & PGN0135-5R-erm-comp & CAATAGCGGAAGCTaactgcgcaaagagcaataat \\
\hline \multirow[t]{2}{*}{ pot-erm } & PGN0135-5F-ermF & ctttgcgcagttAGCTTCCGCTATTGCTTTTTTGC \\
\hline & PGN0135-3R-comp-erm & aacgacaccgaaCTCTAGAGGATCCCCGAAGCTG \\
\hline \multirow[t]{2}{*}{ pot 3' fragment-erm } & erm-3F-PGN0135-3F & GATCCTCTAGAGttcggtgtcgttattttcttc \\
\hline & 3R-comp-PGN0135*5 & ttacttcgctaccegctccagccac \\
\hline
\end{tabular}




\begin{tabular}{|l|l|l|}
\hline \multirow{2}{*}{ sst expression } & PGN1640-5F1*1 & atgcgtaaactacggatcggectct \\
\cline { 2 - 3 } & 3R-PGN1640-comp & cettgcgactgcttgcctcttgcgc \\
\hline \multirow{2}{*}{ opt expression } & 5F-PGN1518 & atggaaaacaaacagacaacgagcat \\
\cline { 2 - 3 } & 3R-PGN1518-comp & tgcttcatgttcttgcctctgatgctgtc \\
\hline \multirow{2}{*}{ pot expression } & 5F-PGN0135 & atgctaaagaatcaccctaaagggttaatctc \\
\cline { 2 - 3 } & 3R-PGN0135-comp & cttcgctaccegctccagccactta \\
\hline
\end{tabular}




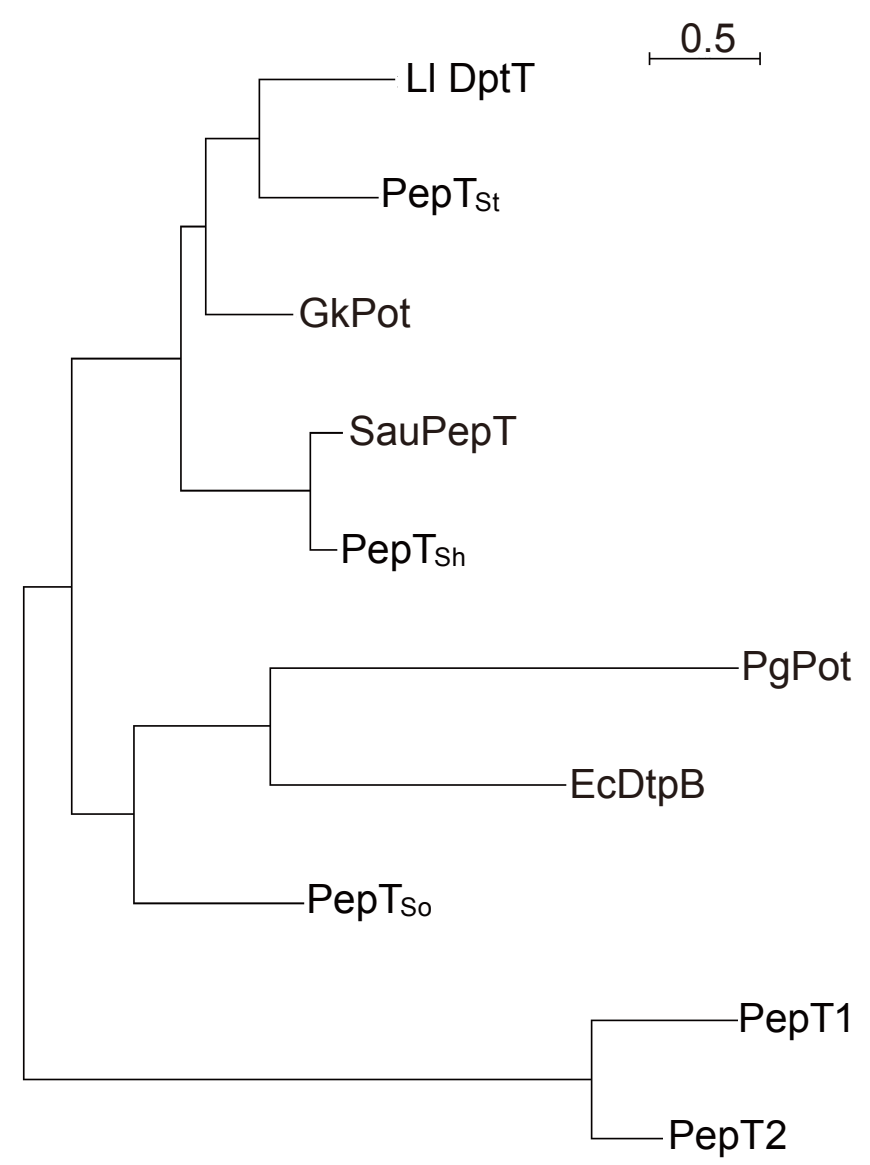

Supplemental Figure S1. Phylogenetic tree of the POT family members.

A phylogenetic tree of $P$. gingivalis POT and bacterial homologues shown in Fig. 4 and human homologues was created using the PhyML package with default settings following sequence alignment with Clustal W. $P$. gingivalis PgPot (UniProt: B2RH09), Staphylococcus hominis PepT $\mathrm{T}_{\mathrm{Sh}}(\mathrm{A} 0 \mathrm{~A} 533 \mathrm{~J} 3 \mathrm{Z}$ ), Staphylococcus aureus SauPepT (A0A0H2XIN4), Geobacillus kaustophilus GkPot (Q5KYD1), Streptococcus thermophilus PepT St $_{\text {t }}$

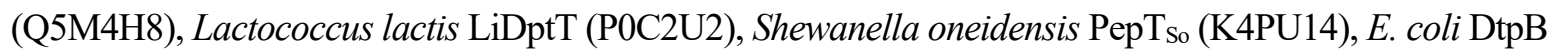
(P36837), Homo sapiens PepT1 (O43641) and PepT2 (S15A2). 